\title{
LA INFLUENCIA DE LA sitcom AMERICANA EN LA PRODUCCIÓN DE COMEDIAS TELEVISIVAS EN ESPAÑA. EL CASO DE "FRIENDS" Y "7 VIDAS"
}

\author{
$M^{a}$ del Mar Grandío Pérez \\ (Universidad Católica San Antonio de Murcia) \\ mgrandio@pdi.ucam.edu \\ Patricia Diego González \\ (Universidad de Navarra) \\ pdiegon@unav.es
}

\begin{abstract}
Resumen: Este artículo tiene como objetivo principal explicar la influencia de la sitcom norteamericana en la producción de comedias televisivas españolas con el fin de obtener los estándares de producción de este formato en España. Se tratará, en concreto, el caso de "Friends" y "7 vidas" por ser el más paradigmático de este fenómeno. La estandarización y profesionalización del proceso creativo así como el alargamiento de la duración serán algunos aspectos característicos tratados en la producción de sitcoms en España.
\end{abstract}

Palabras clave: Televisión, género, sitcom, series, ficción.

Abstract: This article aims to explain the influence of the American sitcom on the production of Spanish television comedies in order to obtain the standards of production of this format in Spain. The case of "Friends" and "7 vidas" will be studied for being the most paradigmatic of this phenomenon in our country. The standardization and professionalization of the creative process as well as the longer duration of this format will be some typical aspects of the production of sitcoms in Spain.

Keywords: Television, genre, sitcom, shows, fiction.

\section{INTRODUCCIÓN}

stos últimos años estamos viviendo una auténtica época dorada de la ficción televisiva tanto por la cantidad como por la calidad de la misma. "Urgencias" (NBC, 1994), "Los Soprano" (HBO, 1999), "El Ala Oeste de la Casa Blanca" (NBC, 1999), "Mujeres Desesperadas" (ABC, 2004) o "House" (Fox, 2004) son solamente algunos éxitos televisivos que son consideradas como televisión popular de calidad. Son series de carácter comercial, que buscan grandes audiencias, que consiguen un notable fenómeno fan en torno a ellas, y que logran asemejarse al cine por la envergadura de producción y el cuidado en la elaboración de sus historias (Jancovich y Lyons: 2003; Mccabe y Akass: 
La influencia de la Sitcom americana en la producción de comedias televisivas en España. El caso de "Friends" y "7 vidas"

2007). La mayoría provienen de Estados Unidos, aunque también encontramos interesantes series domésticas españolas como "Cuéntame" (La 1, TVE, 2001), "Los Simuladores" (Cuatro, 2006) o "Desaparecida" (La 1, TVE, 2007). En España, a lo largo de la década de los noventa, fue cuando la ficción propia demostró la capacidad de liderar el panorama televisivo hasta convertirse en el género favorito del público y uno de los más rentables para las televisiones (Diego y Pardo, 2008: 47).

Pero no son las únicas. "Seinfeld" (NBC, 1989), "Friends" (NBC, 1994) o "Frasier" (NBC, 1993) son algunas de una larga lista de comedias que han marcado el ritmo de la comedia de situación a finales del siglo XX. Alcanzaron su máximo apogeo en la década de los noventa y muchos críticos anunciaron su muerte cuando estas sitcoms clásicas finalizaron sus emisiones. Sin embargo, es un formato televisivo que se ha adaptado perfectamente en otros países fuera de Estados Unidos y que, además, ha revitalizado otros sistemas de producción televisiva tal y como ocurre con el caso español.

Este artículo pretende analizar, precisamente, la influencia que la comedia de situación o sitcom ha tenido en la producción de telecomedias en España con el fin de obtener los estándares de producción del formato de la sitcom en nuestro país. Se tomará como ejemplo el caso de "Friends" y la directa relación que tuvo en la producción de "7 vidas" en nuestro país. "Friends" es una de las comedias más populares de los últimos años que obtuvo un sonado éxito entre crítica y público. Sus diez temporadas en antena y sus numerosos galardones la han encumbrado como una de las mejores comedias de situación de la historia de la televisión. En el caso español, "7 vidas" se ha convertido en la sitcom más longeva de emisión nacional gracias a sus siete años en antena. Pese a no tener unos resultados de audiencia espectaculares en sus inicios, el buen trato de la crítica fue aumentando su seguimiento temporada a temporada.

En primer lugar, en este artículo se explicarán las características principales de la comedia de situación de Estados Unidos para pasar, a continuación, a desarrollar la evolución del género de la comedia en España. En último lugar, estudiaremos propiamente la influencia de "Friends" en la producción de comedias españolas analizando el caso de "7 vidas".

\section{LA COMEDIA DE SITUACIÓN O SITCOM EN ESTADOS UNIDOS}

Las telecomedias han sido siempre uno de los formatos de ficción más prolíficos en la pequeña pantalla. En Estados Unidos este género-comedy- se ha materializado de diferentes formas, como la comedia de situación o sitcom, el sketch show, la comedia de stand-up o los dibujos animados para adultos (adult animation) (Creeber, 2001: 13-113). Sin embargo, probablemente sea la sitcom el formato con mayor tradición, apoyo popular y peso específico dentro de la industria americana de televisión. Algunos autores consideran las comedias de situación como uno de los géneros más convencionales de la televisión estadounidense. 
Originaria de la década de los 50 , nació muy unida a la televisión comercial de este país y, a día de hoy, sigue siendo Estados Unidos el país donde más producción se genera:

Hablar de la comedia de situación norteamericana es pura redundancia. La sitcom es tan norteamericana como la cadena de producción del automóvil Henry Ford. Porque la comedia de situación se ha hecho con sus productores, guionistas, actores, y como tal es un acto creativo, estético, pero con clara dependencia de un sistema televisivo que ha ido funcionando con pocos cambios desde hace 50 años y que marca de forma profunda el desarrollo de este género y de los que con él comparten programación (Álvarez Berciano, 1999: 14).

A pesar del predominio de comedias de situación americanas, no tenemos tampoco que olvidar la importancia del mercado británico en la producción de sitcoms con comedias tan reputadas como "Fawlty Towers" (BBC, 1975), "The Good Life" (BBC, 1975), "Only Fools and Horses" (BBC, 1981) o "Yes Minister" (BBC, 1980). Además, es también notoria la influencia que las sitcoms americanas han tenido en la producción de series domésticas en otros países, como puede ser España tal y como veremos.

Si buscásemos una definición operativa sobre este formato televisivo, encontraríamos que el término sitcom es la abreviatura de situation comedy y se refiere a una serie de media hora de duración en la que los personajes se encuentran involucrados en una situación cómica (Wolf, 1996: 3). Con esta explicación, se puede ya apuntar cómo lo más significativo de las comedias de situación es observar cómo reaccionan los personajes ante los conflictos que se les presentan. De hecho, los personajes intentan escapar de sus problemas cotidianos pero sus expectativas se ven continuamente frustradas (Curtis, 1982: 10-11).

Con una herencia directa del teatro y de los seriales radiofónicos, las comedias de situación han confiado en su artificialidad para conseguir mayor efectividad humorística. Algunos autores hablan incluso de una "transparente artificialidad" (Mills, 2004: 67) representada, principalmente, por el disco de risas enlatadas, la puesta en escena tan teatral o la propia actuación de los actores.

Desde sus orígenes y durante más de 50 años, su modelo de producción y estructura narrativa no ha variado prácticamente llegando a ser, como comentábamos antes, uno de los géneros más inalterables de la parrilla televisiva estadounidense. "I love Lucy" (CBS, 1951), "The Dick Van Dyke" (CBS, 1961), "Hechizada" ("Bewitched") (ABC, 1964), "Murphy Brown" (CBS, 1988) o "Cheers" (NBC, 1982), son sólo algunos ejemplos de sitcoms exitosas que culminaron su época dorada cuando "Seinfield" (NBC, 1990), "Frasier" (NBC, 1993) y "Friends" (NBC, 1994) compartieron "noche de humor" en la NBC. Se detallan a continuación algunas de las características principales de las sitcoms clásicas (Cortés, 2000: 185-189):

1. Es un producto serial a capítulo cerrado, de corta duración (22 minutos aproximadamente) y preparado para una posible larga permanencia en antena. 

comedias televisivas en España. El caso de "Friends" y "7 vidas"

2. Suele pivotar sobre dos o tres personajes, estereotipados, a veces contrarios, sometidos a situaciones corrientes de la vida diaria. Los personajes funcionan con características muy marcadas, si bien virtudes y defectos no están expuestos de manera radical.

3. La grabación se realiza normalmente en interiores y con público en directo. Generalmente utiliza un decorado único, divido en varios sets. Se utilizan tres o cuatro cámaras.

4. El mundo exterior apenas se ve, salvo en contadas ocasiones; sin embargo, está presente siempre a través del diálogo.

5. Dos elementos las caracterizan de forma fundamental a la hora del guión: diálogos cortos, vivos, agudos, muy elaborados, y los gags visuales.

Desde el punto de vista de la realización, la actuación de los personajes es herencia del teatro aunque la grabación con la cámara es televisiva. A este respecto, la realización típica de la sitcom es multicámara, es decir, se utilizan varias cámaras simultáneamente. Otras de las claves de la comedia es la puesta en escena como parte del espectáculo. Hablamos de elementos como el escenario, el vestuario, el movimiento de los actores. En el caso de la comedia, la excelencia en la actuación de los actores es crucial. De hecho, una de las razones del fracaso del humor es una actuación inadecuada. Pero tal vez lo más específico del producto sea el marcaje de los énfasis cómicos con un disco de las risas enlatadas para que cause en el espectador un movimiento reflejo hacia la carcajada.

Sobre la construcción del guión de la sitcom, cabría decir que los principios dramáticos aristotélicos han sido ajustados a la naturaleza del medio televisivo. En primer lugar, en la década de los 80 se añadieron más niveles de narración con las llamadas multistory sitcoms que aumentaron la complejidad a las tramas argumentales y dieron más fuerza dramática a las comedias de situación tradicionales. Por regla general, en las comedias televisivas clásicas como "Friends" hay una media de tres tramas por capítulo, una de ellas la principal y hasta tres subtramas. Por otro lado, nuevos telespectadores puede que sintonicen con el programa en cualquier momento y necesiten información de acontecimientos pasados. Es lo que se denomina redundacy o dispersed exposition que vendría a ser como un personaje que recapitula, normalmente por medio del diálogo, la información que ha ocurrido hasta el momento (Thompson, 2003: 56).

En segundo lugar, la estructura de las comedias de situación depende claramente de su emisión. Cada capítulo se divide en dos partes de 12 minutos separadas por la publicidad. De esta manera, el guión está también partido en dos actos separados por el corte publicitario. Al final de este primer bloque, la acción deberá quedarse en suspense en lo que se llama cliffhanger, es decir, se abre una acción dentro de la trama episódica que no se cerrará hasta después de la publicidad (Thompson, 2003: 42), alimentando la intriga y asegurándose, de esta manera, que el espectador permanezca frente al televisor para conocer 
cómo concluye el capítulo. Además, también existen cliffhangers de temporada a temporada (Thompson, 2003: 62) que se generan al mantenerse una trama central de la serie abierta hasta el primer episodio de la siguiente temporada, algo muy habitual en género dramáticos y que fomenta la fidelización de los espectadores en el tiempo. En este sentido, originariamente la mayoría de las sitcoms fueron concebidas como episodios independientes unos de otros aunque, conforme se iba acentuando el sistema televisivo comercial y la lucha de las audiencias, poco a poco fueron incorporando tramas al estilo de la soap opera a través de tramas seriales para poder mantener los índices de popularidad a través del propio argumento.

En tercer lugar, la mayoría de las sitcoms tienen el denominado teaser o hook al inicio de cada episodio. Es una corta escena que sirve para captar la atención de la audiencia y asegurar que continuará viendo la serie después del primer corte publicitario. En Estados Unidos se coloca habitualmente antes de los títulos de crédito. Normalmente son independientes de las tramas aunque, cada vez más, adelantan alguna de las tramas principales o secundarias del episodio. Por otro lado, al final del capítulo está el denominado tag o cola que se emite durante los títulos de créditos (Rannow, 2000: 30). Es una corta escena que se presenta como chiste final.

Desde el punto de vista de la programación, las sitcoms fueron concebidas como programas de acceso a las franjas de horario televisivo importantes, fundamentalmente de prime time, y, por tanto, fueron llamadas a concentrar un buen número de espectadores a los que arrastran después al programa estrella de la noche. Sin embargo, y gracias a su gran capacidad para mantener fiel a la audiencia, llegaron a convertirse en programas de máxima audiencia, tal y como ocurre con "Friends" (NBC, 1994-2004), entre otras. El público al que se dirigen estos productos audiovisuales se podría decir que es familiar, aunque a raíz de la década de los noventa aparecieron sitcoms dirigidas a grupos más específicos de audiencia, como pueden ser las mujeres o los jóvenes.

A pesar del conservadurismo que ha caracterizado la evolución de la comedia de situación en Estados Unidos, en la actualidad nuevas apuestas revitalizan este formato a día de hoy. Series como "The Office" (BBC, 2001), "Arrested Development" (Fox, 2003), "Curb with Enthusiam" (HBO, 2000) o "Extras" (BBC$\mathrm{HBO}, 2005)$ representan un cambio generacional en las comedias de situación que abren una auténtica innovación formal en el género. Aspectos como la mezcla de ficción y realidad, la influencia del estilo documental o el cine así como la representación de personajes y un humor absurdo rompen formalmente con el clasicismo tan preponderante en este formato durante más de cinco décadas.

\section{LA EVOLUCIÓN DEL GÉNERO DE LA COMEDIA EN ESPAÑA}

Centrándonos ya en España, al género de la comedia se le ha denominado normalmente como telecomedia o series cómicas, antes que usar el término 
La influencia de la Sitcom americana en la producción de comedias televisivas en España. El caso de "Friends" y "7 vidas"

americano sitcom. En nuestro país también han sido abundantes los programas humorísticos basados en sketchs y, sobre todo, la serie cómica o telecomedia. El formato español de telecomedia ha surgido como mezcla de tres géneros diferenciados, a saber: la sitcom; el teatro encarnado en el género del sainete; y la comedia cinematográfica de los años 60,70 y 80 .

Por un lado, las producciones nacionales sí han adaptado una serie de características propias de la sitcom como personajes estereotipados, reducido número de localizaciones interiores, recurso de las risas enlatadas, grabación con público delante, gags como elementos esenciales de la comicidad, y el protagonismo de una estrella alrededor de la cual se articula la serie.

Por otro lado la telecomedia de producción nacional ha tenido grandes influencias de la tradición teatral española, y en especial, del sainete. Este género teatral se puede definir como una breve pieza teatral, cómica y popular, propia del Siglo de Oro hasta mediados del siglo XIX, que aparecía intercalada en los intermedios o entreactos de una obra dramática. Sus características eran la brevedad, la crítica burlesca a la sociedad y a las costumbres y el uso del lenguaje popular (Estébanez, 1996):

Si hoy mismo tuviéramos que hablar de lo sainetesco es muy probable que nos remitiéramos a las numerosas telecomedias nacionales que tan espectacular desarrollo han alcanzado en las últimas temporadas. Algunos de sus guionistas y realizadores -Vicente Escrivá y Sebastián Junyent, por ejemplo- han reconocido explícitamente la influencia del sainete teatral, lo cual no es extraño en unos profesionales que proceden con cierta frecuencia del ámbito teatral o conocen la tradición de un género con importantes paralelismos con los presupuestos de algunas de estas comedias. El resultado es un público televisivo que disfruta con las andanzas cotidianas y domésticas de unos tipos -interpretados por actores teatrales con toda la teatralidad al usopopulares y simpáticos, que mezclan el humor y la ternura en unos ámbitos fácilmente reconocibles y públicos (farmacias, supermercados, barrios...) (Ríos, 1997: 159).

Algunas de las características que Juan Antonio Ríos (Ríos, 1997: 21-22) señala como propias del género sainetesco, pueden aplicarse a muchas telecomedias españolas: regionalismo o localismo cercano a lo folclórico (andalucismo, madrileñismo); personajes que responden a tipos recurrentes o estereotipados; deformación de la lengua con objeto humorístico (empleo de juegos de palabras, dobles significados, etc.); tendencia al melodrama y al sentimentalismo en las tramas y argumentos, etc. Asimismo, se producen numerosas escenas que no hacen avanzar la acción dramática sino que están basadas en el diálogo. Normalmente los finales de estas obras suelen ser felices e incluyen, en muchos casos, una moraleja o un adoctrinamiento. La utilización de chistes y retruécanos es muy frecuente en un ambiente costumbrista para dar una imagen más global de la realidad, donde se da una representación de la clase popular. Otra característica es la idealización de los ambientes sociales sin resaltar los aspectos negativos 
de la realidad, ofreciendo un reflejo amable e ingenuo ya que la finalidad es divertir al espectador.

Finalmente, como tercer influjo, la comedia cinematográfica española de los años 60, 70 y 80 también ha influido en el género televisivo. En la década de los 60, entre otras producciones cinematográficas, se encuentran las llamadas comedias "a la española" o "españoladas" realizadas por directores como Mariano Ozores, Tito Fernández, Pedro Lazaga o Fernando Palacios. Algunos títulos de comedias destacados fueron: "La ciudad no es para mí" (1965) de Pedro Lazaga, "La gran familia" (1962) de Fernando Palacios o "Cómo está el servicio" (1968) de Mariano Ozores. Normalmente se trataba de comedias dirigidas al gran público, con un humor poco refinado, insistente en alusiones sexuales o de carácter machista y en la satirización de personajes como el paleto o "cazurro", el "reprimido", la española de "origen humilde", los turistas, los ejecutivos norteamericanos de empresas multinacionales o un tratamiento caricaturizado de la mujer (Torreiro, 1995: 333). Algunas de ellas fueron interpretadas por actores y actrices de renombre como Paco Martínez Soria, José Luis López Vázquez, Gracita Morales, Antonio Ozores, Concha Velasco, José Sacristán o Alfredo Landa.

Prueba de esta unión entre las "españoladas" y las telecomedias es que muchos actores y actrices que trabajaron en estas comedias han protagonizado series en la pequeña pantalla, como por ejemplo: "Lleno por favor" (Antena 3, 1993), "Por fin solos" (TVE 1, 1995) y "En plena forma" (Antena 3, 1997) con Alfredo Landa; "¿Quién da la vez?" (Antena 3, 1995) y "Éste es mi barrio" (Antena 3, 1996) con José Sacristán; "El sexólogo" (TVE 1, 94-95) con Antonio Ozores; "Yo, una mujer" (Antena 3, 1995) y la primera temporada de "Compañeros" (Antena 3, 1998) con Concha Velasco; "Los ladrones van a la oficina" (Antena 3, 1993) con Fernando Fernán Gómez, José Luis López Vázquez, Agustín González y Manuel Aleixandre; "Makinavaja" (TVE 1, 1995), "¡Ay, Señor, Señor!" (Antena 3, 1994) y "Tío Willy" (98) con Andrés Pajares; Lina Morgan con "Compuesta y sin novio" (Antena 3, 1994) y "Hostal Royal Manzanares" (TVE 1, 1995); Arturo Fernández con "Truhanes" (Tele 5, 1995) y "La casa de los líos" (Antena 3, 1996).

Éste fue el origen de un tipo de telecomedias creadas para ser "vehículos de estrellas", es decir, constituidas alrededor de actores y actrices veteranos dentro del panorama audiovisual español, tal y como destaca García de Castro (2002: 123):

La vocación de comedia de situaciones motivó en un principio la importancia del star-system en todas estas series. Se consideraba que los protagonistas populares determinaban el éxito de la producción. Se pensaba en una serie para un protagonista y no para una idea. Las comedias de situación de esos años buscaron una estrella y descuidaron el resto.

De igual modo encontramos guionistas, realizadores y productores que iniciaron su trayectoria profesional cultivando este género en el ámbito cinematográfico para hacerlo luego en televisión. Tal es el caso del guionista, director y productor 
La influencia de la Sitcom americana en la producción de comedias televisivas en España. El caso de "Friends" y "7 vidas"

Vicente Escrivá, artífice de películas emblemáticas de corte religioso y político en los años 50, y gran promotor de la comedia "a la española" durante los años 60 , 70 y 80 como "Los ladrones somos gente honrada" (1956) o "Cateto a babor" (1970). En la década de los 90 dirige para Antena 3 algunas de sus primeras series, antes mencionadas, como "Lleno, por favor", “¿Quién da la vez?", "Éste es mi barrio" y "Manos a la obra". Otro tanto podría afirmarse de José Frade, prolífico productor de telecomedias con un gran currículum cinematográfico, a quien se deben series como "Hermanos de leche" (1994) y "Canguros" (1994) para Antena 3.

Desde importantes foros de análisis de la ficción televisiva española, profesionales involucrados en la creación y producción de series cómicas han calificado estas series como productos autóctonos e híbridos, consecuencia de la extrapolación de la sitcom a la realidad cultural de España. Así lo afirma, entre otros, el guionista y productor de series y programas, Tom Roca (1995: 64-65):

Creo que hay un principio de la sitcom clásica que sí está funcionando en España. Esta es la base de que estas comedias, sean o no sitcom, signifiquen un formato extraño que nos hemos inventado, de 45 ó 50 minutos. Lo que sí tienen como base es que los personajes resultan entrañables, resultan creíbles y las situaciones son próximas a nuestra realidad cotidiana [...]. Creo que muchas producciones que se nos han presentado como comedias de situación han acabado siendo dramas lacrimógenos. Yo no sé qué género es este. El "tempo" de una sitcom son los veintidós minutos, la media hora comercial. Esto se hace en Estados Unidos y en Inglaterra hace cincuenta años, aquí las hacemos de cuarenta y cinco minutos y a mí se me pierde un poco el esquema. Claro, llegamos a la improvisación, creo que en España improvisamos, salvo honrosos ejemplos [...]. También me remito a la frase de un amigo mío que me dijo "mira, aquí llevamos cuatro o cinco años de televisión privada y en Estados Unidos llevan cincuenta".

Otros autores como García de Castro (2002: 121) reconocen también la particular adaptación que la industria nacional ha realizado de la comedia de situación americana, sobre todo en su duración:

En España no se implanta inicialmente la comedia de situación pura sino una adaptación local, una telecomedia de carácter costumbrista, que incluye una mezcla de géneros. Así pues, la versión española introduce variables específicas. Algunas de estas series tienen una duración superior a los 30 minutos, lo que supone una novedad del formato español. Esta ampliación del tiempo de su emisión está provocada por la duración del prime time y la necesidad de su rentabilización comercial, lo que hizo que se introdujeran componentes del drama para mantener la atención del público.

Algunos de estos rasgos teatrales y cinematográficos se observan también en telecomedias como "Farmacia de guardia" (Antena 3, 1991), "Los ladrones van a la oficina" (Antena 3, 1993), "Compuesta y sin novio" (Antena 3, 1994), "iPor fin solos!" (TVE 1, 1995), "Villarriba y Villabajo" (TVE 1, 1994) u "Hostal Royal Manzanares" (TVE 1, 1996). 


\section{LA INFLUENCIA DE "FRIENDS" EN LA PRODUCCIÓN DE COMEDIAS ESPAÑOLAS. EL CASO DE "7 VIDAS"}

Aunque en menor número, también se han producido en España series con influencia de la sitcom americana en su estructura dramática y en la aplicación de método de producción americano. La serie que inaugura este tipo de sitcoms "a la española" es "7 vidas" (1999), tipo "Friends". Otros ejemplos son "iAla...Dina!" (TVE 1, 2000), que recuerda a la serie americana "Embrujada (Bewitched)", o "Moncloa ¿dígame?" (TVE 1, 2001), inspirada en la sitcom americana "Spin City". En este artículo estudiaremos la especial relación que se estableció entre la comedia de situación americana "Friends" y la telecomedia española "7 vidas".

Se podría clasificar "Friends" como una de las comedias de situación clásica más exitosas de los últimos tiempos en Estados Unidos y en todo el mundo. Producida por la productora Warner Bross y emitida originariamente por la cadena norteamericana NBC, cosechó grandes éxitos de audiencia desde sus primeros episodios en 1994. Esta serie contaba de manera cómica las peripecias de varios amigos veinteañeros en Nueva York. Tras diez temporadas consecutivas en pantalla y 230 episodios, alcanzó una gran popularidad dentro y fuera de sus fronteras (Grandío, 2007) así como el respeto de los críticos. En concreto, recibió 55 nominaciones a los premios Emmy y ganó el Emmy a Mejor Comedia en 2003. Con una media de 25 millones de audiencia, su episodio final alcanzó los 56,2 millones de telespectadores, colocándose así como el cuarto final de serie más visto de toda la historia de la televisión en Estados Unidos por detrás de "MASH" (CBS, 1970), "Seinfeld" (NBC, 1990) y "Cheers" (NBC, 1982) según los datos recogidos por la empresa de medición de audiencias Nielsen ${ }^{2}$. Es llamativo destacar cómo los cuatro episodios finales más vistos de todos los tiempos en Estados Unidos sean sitcoms clásicas, algo que demuestra la buena acogida que este género televisivo ha tenido en el país donde se originó. Tras las diez temporadas en antena, en 2004 se creó un spinoff de "Friends" con el nombre de uno de sus protagonistas, "Joey" (NBC, 2004), que tan sólo duró 3 temporadas en la parrilla.

Digno de mención son también los elevados presupuestos destinados a la realización de las comedias de situación en Estados Unidos. Según la revista Forbes, el coste medio de producción de un capítulo de una sitcom en Estados Unidos asciende a 1.300.00 dólares (Forbes, 2003), a lo que hay que sumar costes como los generados por la contratación del reparto en "Friends", cuyos actores recibieron durante las últimas dos temporadas un millón de dólares por cada capítulo grabado, convirtiéndose así en la telecomedia más cara de la historia de la televisión (El Mundo, 2004).

En España, y a pesar de sus emisiones a través del canal de pago Canal +, "Friends" también tuvo muchos seguidores desde 1997, llegando incluso a

En 1983, "MASH" consiguió 105,4 millones. En 1993, "Cheers" alcanzó 80,3 millones de espectadores y "Seinfeld" 76,2 millones en 1994. Los datos provienen de Nielsen Media Research. 
La influencia de la Sitcom americana en la producción de comedias televisivas en España. El caso de "Friends" y "7 vidas"

generarse un gran fenómeno fan en torno a la serie. Han sido numerosas las reposiciones en abierto de esta serie en Canal $+y$ posteriormente en Cuatro. Muchos de los seguidores, al intentar explicar por qué les gustaba la serie, llegan a afirmar que "Friends" podría considerarse una serie "de gran calidad" o, incluso, una comedia "de culto" (Grandío, 2009), calificación que el mundo académico ha destinado al material de Ciencia Ficción casi exclusivamente. Estamos, por tanto, ante una serie que ha marcado un hito en relación con el fenómeno fan generado en nuestro país, algo que pudo motivar también el interés que la productora Globomedia tuvo en desarrollar un proyecto parecido en España.

En este sentido y como comentan algunos seguidores españoles, es cierto que "Friends" ha alcanzado la excelencia en los estándares de producción de la comedia de situación clásica. El equipo de guionistas capitaneado por Marta Kauffman y David Crane y el productor Kevin Bright crearon todo tipo de tramas siempre dentro del esquema clásico de comedia de situación. Aunque con grandes influencias de la soap opera, sobre todo relacionadas con las tramas amorosas entre los personajes, nos encontramos sin lugar a dudas ante una sitcom de corte tradicional basada en personajes estereotipados, episodios conclusivos de 22 minutos y un sistema de producción multicámara grabado en interiores principalmente. Un bolso, un mono, una cena de Acción de Gracias o el cameo de un invitado especial (desde Julia Roberts hasta George Clooney) han sido el centro de tramas cómicas.

Sin embargo, el legado de "Friends" es incluso mayor que su propio éxito y, como comentamos, ha tenido también su repercusión en la producción de series domésticas en distintos países. En todo el mundo aparecieron pronto series que imitaban o se inspiraban en la popular serie norteamericana. Por su parte, en nuestro país se han producido series nacionales siguiendo el esquema de "Friends" como "Más que amigos" (1997), aunque el caso más claro sea tal vez "7 vidas" (1999). Tratemos en concreto el caso de la influencia de "Friends" en esta comedia española.

"7 Vidas" es una serie producida por Globomedia, una de las más importantes productoras de ficción españolas. Se origina en 1999 con la idea de convertirse en la primera sitcom propiamente dicha de la ficción nacional que adoptara, como hemos mencionado, su estructura narrativa y el proceso de producción. Un año antes, "Fernández y familia" (Tele 5, 1998) y "Quítate tú pa' ponerme yo" (Tele $5,1998)$ fueron dos intentos fallidos de producciones de este tipo. La influencia del gran éxito mundial de "Friends" está presente en "7 vidas" desde el origen mismo de la idea. La premisa o conflicto que resume la idea de la producción nacional era muy similar a la de "Friends". Los guionistas de esta serie dejaban clara su influencia desde su inicio:

Escribir sobre una pandilla de amigos treintañeros que vivían en un entorno urbano. Sí, vale como "Friends" pero con la pequeña diferencia de que en lugar de Ross o Chendler, nosotros teníamos a Paco Jimeno, algo más... autóctono (Pastor y de Pando, 2006: 26). 
Otro dato bastante indicativo de la influencia de "Friends" en la producción española era que una de las pruebas que Globomedia tenía para contratar guionistas para la serie consistía en escribir una secuencia de "Friends". La serie de la NBC se convirtió en referente esencial de "7 vidas" (Pastor y de Pando, 2006; 43). La dinámica de escritura de los capítulos se importa del sistema americano de escritura por equipos. Había unos doce guionistas divididos en equipos de tres y cada equipo contaba con la presencia de un jefe, un segundo y un guionista junior y una media de mes y medio de trabajo por episodio. Cuando contaban con una versión más definitiva del guión, al igual que se hace en el proceso de producción americano, tenía lugar la Writer's room. Los guionistas se encierran todos juntos a pensar gags para añadir comicidad a los guiones que después en la "Mesa italiana", de boca de los actores, se comprueba si funcionan en el guión. Este método de escritura por equipos no estaba implantado en España ya que la mayoría de la series trabajaban con guionistas independientes o freelances que trabajan de forma individual los guiones de los capítulos.

Como hemos mencionado, "7 vidas" importa la estructura narrativa de la sitcom, pero con una particularidad: la adapta al tiempo medio, 50-55 minutos por capítulo, a diferencia de los 25 de la americana. De esta manera, el teaser, primer acto, segundo acto, desenlace y tag quedan alargados de manera a veces un poco forzada.

Desde el 17 de enero de 1999 hasta el 16 de abril de 2006 se emitieron 204 capítulos de "7 vidas" repartidos en 10 temporadas con algunos hitos para la producción de comedias en España. Por ejemplo, el 12 de marzo de 2006 se celebró su capítulo número 200 con una emisión en directo en la que participó parte del elenco original junto con el de la última temporada. Se convertía, de este modo, en la primera emisión en directo de una serie de televisión de estas características.

El proceso de producción es una réplica del estándar americano planificado en 5 días. A continuación ofrecemos el método de trabajo aplicado por la productora: 
La influencia de la Sitcom americana en la producción de comedias televisivas en España. El caso de "Friends" y "7 vidas"

Tabla 1. Proceso de producción de los capítulos de "7 vidas"

\begin{tabular}{|c|c|c|}
\hline \multirow[t]{4}{*}{$1^{\circ}$ día: } & $9: 30$ & $\begin{array}{l}\text { Lectura de guión: el director comenta su visión del guión } \\
\text { y cada miembro de los diferentes equipos desglosa sus } \\
\text { necesidades. }\end{array}$ \\
\hline & $10: 30$ & $\begin{array}{l}\text { Mesa italiana: los actores junto con el director realizan una } \\
\text { lectura del guión. }\end{array}$ \\
\hline & $11: 30$ a $14: 30$ & $\begin{array}{l}\text { Ensayo leído en decorados: se organiza una puesta en } \\
\text { escena de los actores, el director marca sus posiciones y } \\
\text { movimientos. Este ensayo se realiza de manera cronológica } \\
\text { y sin la presencia del equipo técnico. }\end{array}$ \\
\hline & $15: 00$ & $\begin{array}{l}\text { Ensayo del episodio para la producción ejecutiva: se ensaya } \\
\text { el capítulo con texto y movimientos para que el productor } \\
\text { ejecutivo otorgue el visto bueno y proponga cambios. }\end{array}$ \\
\hline $2^{\circ}$ día: & $9: 00$ a $14: 00$ & $\begin{array}{l}\text { Puesta en escena: los actores ya tienen el guión } \\
\text { memorizado y el ensayo se realiza sin vestuario ni } \\
\text { maquillaje. }\end{array}$ \\
\hline $3^{\circ}$ día: & $9: 30$ a $18: 30$ & $\begin{array}{l}\text { Ensayo técnico: se realiza un ensayo completo por } \\
\text { localizaciones, no cronológico, con todos los equipos } \\
\text { técnicos y los actores con su vestuario, maquillaje y } \\
\text { peluquería. Se graban las escenas que son imposibles de } \\
\text { realizar en directo. }\end{array}$ \\
\hline \multirow[t]{2}{*}{$4^{\circ}$ día: } & $10: 30$ a $\quad 14: 30$ & $\begin{array}{l}\text { Ensayo general: se reproduce el capítulo cronológicamente } \\
\text { como si se estuviera grabando pero sin público delante. }\end{array}$ \\
\hline & $16: 30$ a $22: 00$ & $\begin{array}{l}\text { Grabación del capítulo con público delante: unas } 100 \\
\text { personas que situadas en unas gradas enfrente de los sets } \\
\text { asisten a la interpretación de los actores. }\end{array}$ \\
\hline $5^{\circ}$ día: & & Edición y sonorización el capítulo \\
\hline
\end{tabular}

Fuente: Elaboración propia con datos de Globomedia.

Otra similitud con "Friends" fueron los cameos de la serie. Más de 100 son los personajes famosos que han pasado por la serie de todo tipo de ámbito profesionales, políticos, deportistas, periodistas, bailarines, etc. El primer cameo se produjo en el capítulo 10 con la aparición de la actriz Lydia Bosch. Algunos otros han sido Santiago Carrillo, Santiago Segura, Antonio Gala, Samuel Eto'o, David Bisbal, Alfonso Guerra, Miguel Ríos, Fernando Torres, Carlos Herrera o Shakira, etc.

También en el área de Dirección Artística de la serie, en el ámbito concreto de los decorados, existía mucha semejanza con "Friends". Las localizaciones 
fijas de la serie, construidas en plató, dan vida a dos salones donde se produce la mayoría de las tramas a los que se une el bar de uno de los protagonistas (Casi Ké No) que incluía, en las primeras temporadas, el típico sofá al más claro estilo "Friends".

"7 vidas" se convirtió en una serie coral por la que desfilaron muchos personajes que fueron cambiando, evolucionando y creando nuevas relaciones entre los que ya estaban. Se convirtió, hasta el momento, en la sitcom más exitosa y longeva de la televisión nacional respaldada siempre por unas buenas cifras de audiencia. Tal es así que, al igual que "Friends" con "Joey" (NBC, 2004) ha dado lugar al spin-off "Aída" (Tele 5, 2005). Ficción que hereda el sistema de producción de "7 vidas", incluso gran parte de su equipo artístico técnico es el mismo y está avalado por el éxito de la audiencia en su sexta temporada.

Para terminar, debemos señalar que a partir de producciones como "7 vidas" casi todas las comedias-sitcom españolas han heredado los siguientes estándares que se han implantado en la industria española y que están presentes en casi todas ellas. Se pueden resumir en las siguientes: duración entre 45 y 70 minutos, soporte vídeo betacam, 13 episodios de media emitidos por temporada, 26 episodios producidos anualmente, emisión semanal, localizaciones $100 \%$ en plató salvo excepciones, entre 2 y 6 decorados fijos, trama organizada en torno a un actor reconocido, aunque cada vez más protagonismo coral.

\section{CONCLUSIONES}

A modo de conclusión, debemos señalar cómo la influencia de la sitcom americana en la producción de comedias televisivas españolas ha sido bastante positiva, tal y como representa el caso de "7 vidas". Su repercusión se ha notado en una doble esfera: en la propia estructura narrativa y en el proceso de producción. El género de la sitcom va asentándose en España como formato de éxito gracias también a "Aída", el spin-off de "7 vidas" que, con sus cinco temporadas, se ha convertido en una de las series con mayor audiencia de las parrillas nacionales.

Debido a su programación en horario de máxima audiencia, la sitcom española ha alargado su duración hasta los 50-70 minutos, muy distante de los 22 minutos de la comedia de situación norteamericana. Respecto a la producción, las telecomedias españolas no cuentan con los altos presupuestos que manejan las grandes productoras y cadenas norteamericanas. En España, el coste medio de producción de una sitcom se encontraría alrededor de los 150.000 euros, mientras que en Estados Unidos sería 1.3 millones de dólares. Lo más positivo de esta influencia es que gracias a la implantación de la sitcom americana en nuestro país, el proceso de producción de las telecomedias se ha estandarizado y profesionalizado. Además, los creativos españoles han adaptado los temas universales de la ficción televisiva a situaciones y personajes típicamente españoles llegando a fidelizar fácilmente, de esta manera, a la audiencia. 
La influencia de la Sitcom americana en la producción de comedias televisivas en España. El caso de "Friends" y "7 vidas"

Dejando de lado antiguos clasicismos formales, el reto que le queda ahora a la industria televisiva española es asimilar también la renovación que se está viendo en la actualidad en el género de la comedia. Las nuevas tendencias en la comedia del siglo XXI afectan a la propia estructura narrativa y a la construcción del humor, aunque uno de los factores más interesantes se centraría precisamente en el ámbito de la realización y de la producción. La industria televisiva española actual ha alcanzado, además, un nivel de madurez para poder aportar ideas innovadoras en el mercado internacional en esta fase de cambio en la que se ven inmersas. Estas nuevas evoluciones del género en nuestra industria convivirán, a su vez, en armonía con las comedias más tradicionales que se alimentan principalmente de la tradición cinematográfica y teatral. Entre ellas podemos destacar "La que se avecina" (Tele 5, 2008) o la malograda "A ver si llego" (2009) retirada de la emisión por Tele 5 sin completar la primera temporada.

\section{REFERENCIAS BIBLIOGRÁFICAS}

ÁlVAREZ BERCIANO, R. (1999): La comedia enlatada. De "Lucille Ball" a "Los Simpson". Barcelona: Gedisa.

CORTÉS, J. A. (2000): La estrategia de la seducción. La programación en la neotelevisión. PAMPLONA: EUNSA.

CREEBER, G. (2000): The television genre book. London: British Film Institute.

CURTIS, B.: Aspects of sitcom. En AA.VV. (1982): Television Sitcom. London: British Film Institute, pp. 10-11.

DIEGO, P.; Y PARDO, A.: Estándares de producción de "dramedias" familiares en España. El caso de Médico de familia, Cuéntame cómo pasó y Los Serrano. En MedinA, M. (2008): Series de televisión. Madrid: Yumelia Textos, pp. 45-74.

EL MUNDO: "La última cita en el Central Perk". 8 de mayo de 2004.

ESTÉBANEZ CALDERÓN, D. (1996): Diccionario de términos literarios. Madrid: Alianza Editorial.

FORBES: "Sex, Money and Videotapes". 17 de marzo de 2003.

GARCÍA DE CASTRO, M. (2002): La ficción televisiva popular. Una evolución de las series de televisión en España. Barcelona: Gedisa y ATV.

GRANDíO, M. (2009): Audiencia, fenómeno fan y ficción televisiva. El caso de "Friends". Buenos Aires: Libros en Red.

-: ¿A qué se debe el éxito de "Friends" en España? Las claves de un humor que ha cruzado fronteras? En CASCAJOSA, C. (2007): La caja lista: televisión norteamericana de culto. Barcelona: Alertes, pp. 35-48.

JANCOVICH, M.; y LYons, J. (2003): Quality Popular Television. Londres: British Film Institute.

MACCABE, J.; Y AKKAS, K. (2007): Quality TV: Contemporary American television and beyond. Londres: I.B. Tauris. 
MILLS, B.: "Comedy Vérité: Contemporary Sitcom Form". Screen, Vol. 45, n² 2, pp. 63-78. PASTOR, S.; Y DE PANDO, C. (2006): 7 años de 7 vidas. Madrid: La esfera de los libros.

RANNOW, J. (2000): Writing Television Comedy. New York: Allworth Press.

Ríos CARRATALÁ, J. A. (1997): Lo sainetesco en el cine español. Alicante: Publicaciones de la Universidad de Alicante.

ROCA, T.: Comedia de situación. En AA.VV. (1995): Ficción televisiva: series. Madrid: Espacio SGAE Audiovisual, pp. 59-69.

THOMPSON, K. (2003): Storytelling in Film and Television. Cambridge: Harvard University Press.

WOLFF, J. (1996): Successful Sitcom Writing. New York: St Martin's.

\section{Breve semblanza biográfica de las autoras}

María del Mar Grandío es profesora de Publicidad y Programación Audiovisual en la Universidad Católica de Murcia (UCAM). Doctora por la Universidad de Navarra, centra su investigación actualmente en el contenido y recepción de las series de televisión. Es autora de Audiencia, fenómeno fan y ficción televisiva (Libros en Red, 2009) y colaboradora en otras publicaciones. Además, ha investigado en Los Ángeles y Gales y ha sido profesora invitada en universidades de Austria, Bélgica, Finlandia y Portugal para hablar sobre series de TV.

Patricia Diego González es profesora de Producción de ficción televisiva y de Programación televisiva en la Universidad de Navarra. Es doctora en Comunicación por la misma universidad en la que recibió el Premio Extraordinario de doctorado por la tesis "La producción de ficción televisiva en España (1990-2002): Evolución histórica, industria, mercado". Ha sido investigadora visitante en la Universidad de Westminster y actualmente ejerce como Subdirectora del Máster en Gestión de Empresas de Comunicación. Su investigación se centra en la producción, industria y mercado de la ficción televisiva y ha colaborado en diferentes libros y artículos sobre la materia.

(Recibido el 10-11-2008, aceptado el 02-03-2009) 